\title{
Aspectos Sociodemográficos e Espaciais dos Casos de Tuberculose Drogarresistente no Estado de Pernambuco
}

\author{
Sociodemographic and Spatial Aspects of Cases of Drug-Resistant \\ Tuberculosis in the State of Pernambuco
}

\section{RESUMO}

Objetivo: Descrever os aspectos sociodemográficos e a distribuição espacial dos casos de tuberculose drograrresistente (TBDR) no Estado de Pernambuco, Brasil, entre os anos de 2015 e 2018. Metodologia: Trata-se de um estudo epidemiológico descritivo, com utilização de dados secundários do Sistema de Informações de Tratamentos Especiais de Tuberculose. Foram analisadas as variáveis sociodemográficas e o padrão de adoecimento dos pacientes notificados. Para análise espacial foi construído mapas temáticos e gráficos demonstrando o coeficiente de incidência anual dos casos de tuberculose drogarresistente em cada município. Resultados: Foram identificados 299 pacientes, sendo $67,3 \%$ do sexo masculino, $80,9 \%$ de cor não branca, $25,7 \%$ com idade entre 21 e 30 anos, $58,5 \%$ com menos de sete anos de estudos e $37,7 \%$ desempregados. Quanto ao padrão de adoecimento: $38,5 \%$ dos pacientes apresentaram inicialmente resistência a rifampicina, $70,2 \%$ foram classificados como casos novos e $29,7 \%$ abandonaram o tratamento. Os maiores coeficientes de incidência estiveram concentrados em municípios situados na faixa litorânea do estado. Conclusão: Foi possível observar uma relação entre os aspectos sociodemográficos e a distribuição dos casos da tuberculose drogarresistente, cuja maior concentração foi na região metropolitana do Estado de Pernambuco. Espera-se que este estudo sirva de subsídio para mediar ações de controle e vigilância da doença.

\section{DESCRITORES}

Tuberculose. Tuberculose Resistente a Múltiplos Medicamentos. Análise Espacial. Saúde Pública. Brasil.

\begin{abstract}
Objective: Describe the sociodemographic and spatial distribution aspects of drug-resistant tuberculosis cases in the state of Pernambuco, Brazil, between the years of 2015 and 2018. Methodology: Descriptive study, with secondary data from the Special Tuberculosis Treatment Information System. The sociodemographic variables and the pattern of illness of the notified patients were analyzed. For spatial analysis, thematic maps and graphs were constructed showing the annual incidence coefficient of drug-resistant tuberculosis cases in each municipality in the state. Results: 299 patients were identified, $67.3 \%$ male, $80.9 \%$ non-white, $25.7 \%$ aged between 21 and 30 years, $58.5 \%$ with less than seven years of formal education and $37.7 \%$ were unemployed. In the pattern of illness: $38.5 \%$ initially presented resistance to rifampicin, $70.2 \%$ were new cases, and $29.7 \%$ abandoned the treatment. The highest incidence coefficient was concentrated in municipalities located along the state coast. Conclusion: It was possible to observe a relation between the sociodemographic aspects and the distribution of cases of drug-resistant tuberculosis, which had the highest concentration in the state's metropolitan region. It is hoped that this study will serve as a subsidy to mediate actions for disease control and surveillance.
\end{abstract}

\section{DESCRIPTORS}

Tuberculosis. Multiple Drug Resistant Tuberculosis. Spatial Analysis. Public Health. Brazil.

${ }^{1}$ Enfermeira, Universidade de Pernambuco, Faculdade de Enfermagem Nossa Senhora da Graças, Recife, Pernambuco, Brasil.

${ }^{2}$ Acadêmica de Enfermagem, Universidade de Pernambuco, Faculdade de Enfermagem Nossa Senhora da Graças, Recife, Pernambuco, Brasil.

${ }^{3}$ Doutora, Pesquisadora da Fundação Oswaldo Cruz - Instituto Aggeu Magalhães, Cidade Universitária, Recife, Pernambuco, Brasil.

${ }^{4}$ Mestre, Docente da Universidade de Pernambuco, Faculdade de Enfermagem Nossa Senhora da Graças, Recife, Pernambuco, Brasil.

${ }^{5}$ Doutora, Docente da Universidade de Pernambuco, Faculdade de Enfermagem Nossa Senhora da Graças, Recife, Pernambuco, Brasil. 
A Tuberculose (TB) é uma doença infectocontagiosa causada pelo Mycobacterium tuberculosis, podendo se manifestar na forma pulmonar, extrapulmonar ou de ambas as formas simultaneamente. Tem distribuição universal e seu risco de adoecimento está relacionado tanto a fatores endógenos, ligados ao sistema imunológico e a determinadas condições clínicas, como também a fatores exógenos como determinantes sociais e condicionantes da saúde. Devido a sua ampla transmissibilidade e por ter profundas raízes sociais, é uma doença que traz grande peso para os serviços de saúde ${ }^{1,2}$.

Sabe-se que a TB está relacionada à pobreza e ao baixo desenvolvimento socioeconômico. Populações pauperizadas, socialmente desfavorecidas e marginalizadas sofrem mais os efeitos da carga de doenças diversas, dentre as quais a TB. Autores relatam que a TB e a pobreza se reproduzem em um ciclo vicioso, onde tanto a existência de más condições de vida propicia o adoecimento, como esta pode produzir a pobreza, quando limita as oportunidades de trabalho ${ }^{3,4}$.

Este quadro social problemático, aliado à avaliação deficiente dos pacientes, à prescrição inadequada de regimes de tratamento e à falta ou falha na provisão e distribuição dos medicamentos padronizados favorece o desenvolvimento de resistência às drogas de primeira linha, o que é tido como um adendo à ameaça ao controle desta enfermidade no mundo. A TB Drogarresistente (TB-DR), que implica em resistência aos dois principais medicamentos de tratamento da doença (rifampicina e isoniazida) é posta, portanto, como grave problema de saúde pública, uma vez que além de dificultar a prevenção e tratamento da doença, contribui para o aumento da mortalidade e perpetuação do quadro epidemiológico ${ }^{1-4}$.

A classificação da TB-DR é dada de acordo com o nível de resistência do bacilo aos tuberculostáticos, podendo ser: resistente apenas a rifampicina $(\mathrm{R})$; monorresistente quando existe insensibilidade à apenas um fármaco antituberculose que não seja a $R$; polirresistente quando é insensível a dois ou mais tuberculostáticos (exceto associação $R$ e Isoniazida $-\mathrm{H}$ ); multirresistente (TB-MDR) quando não respondem a pelo menos à $\mathrm{R} \mathrm{e} \mathrm{H}$ e por fim extensivamente resistente (TB-XDR) quando é insensível a pelo menos à $\mathrm{R}$ e $\mathrm{H}$, à qualquer fluoroquinolona e a pelo menos um dos três medicamentos injetáveis de segunda linha ${ }^{1-4}$.

A Organização Mundial de Saúde (OMS) em 2017 estimou 282 mil novos casos de TB nas Américas, sendo que em um grupo de 30 países responsáveis por $80 \%$ dos casos de TB, o Brasil ocupa a vigésima posição neste ranking, em números absolutos ${ }^{5}$. $O$ cenário brasileiro evidenciou, ainda, uma média de 88.742 casos notificados por ano no período entre 2015 e 2018, totalizando 354.968 notificações de TB. Este número não se distribui equitativamente no território brasileiro, de modo que 93.147 destes concentram-se na região Nordeste, fazendo-o ocupar o segundo lugar no ranking das regiões com maior número absoluto de casos. Pernambuco é o estado do Nordeste com maior número dos casos da doença com cerca de 23.286 confirmados na região ${ }^{6}$.

Considerando o número de casos, políticas com o objetivo de eliminar a TB vem sendo publicadas desde 1993 pela OMS, 
quando também esta doença foi declarada emergência mundial com a recomendação do Directly Observed Treatment Short-Course (DOTS) como estratégia padrão para controle da TB. O Brasil, como signatário, adotou a estratégia e em 2000, alinhando-se aos objetivos da OMS, comprometeu-se a combater a extrema pobreza e outros males da sociedade, dentre eles a TB, até $2015^{7}$.

Em 2014, na Assembleia Mundial de Saúde, ficou aprovada a Estratégia pelo Fim da Tuberculose, tendo como ator principal o Brasil. A estratégia tem como um dos objetivos, o fim da epidemia global da doença e como metas para serem cumpridas até 2035 a redução do coeficiente de incidência para menos de 10 casos por 100 mil habitantes e a redução do número de óbitos por TB em $95 \%{ }^{8}$. Apesar da mortalidade por TB ter reduzido no mundo desde que estratégias para seu controle foram implementadas, 1,7 milhão de pessoas morreram da doença em 2016. Além disso, 5000 pessoas morrem de TB a cada dia, o que contribui para sua elevada carga. Dentre os 22 países que contribuem para $80 \%$ da carga mundial da TB, o Brasil ocupa a $16^{\mathrm{a}}$ posição ${ }^{9,10}$.

Dentre os estados brasileiros que se destacam no controle da TB com relação à adesão às estratégias de controle, Pernambuco avança quando cria, em 2011, o Programa para Enfrentamento às Doenças Negligenciadas (SANAR) que teve sua principal estratégia voltada para a avaliação e monitoramento das metas de eliminação da TB. Essa estratégia permite de forma sistemática apresentar a relação entre as intervenções e os resultados esperados organizado por componentes, atividades, resultados intermediários e impactos, assim como verificar o cumprimento periódico de metas facilitando, assim, o planejamento para ações de controle da TB ${ }^{11}$.

Em detrimento da existência de estratégias específicas para o enfrentamento da TB no Estado de Pernambuco (PE), o histórico de abandono do tratamento ainda é o principal fator de ameaça às medidas de controle ${ }^{12,13}$. De acordo com a resistência, PE esteve, em 2012, entre as cinco unidades federativas com mais de 4 mil casos da TB-DR, sendo responsável por $55 \%$ da carga da doença no país ${ }^{13-15}$.

Considerando a magnitude de TB-DR em Pernambuco e a carência de artigos sobre o tema voltado para o estado, este estudo tem como objetivo descrever os aspectos sociodemográficos e a distribuição espacial dos casos de TB-DR no Estado de Pernambuco, Brasil (BR), entre os anos de 2015 e 2018.

\section{METODOLOGIA}

Estudo epidemiológico de cunho observacional e descritivo, utilizando-se de dados secundários do Sistema de Informações de Tratamentos Especiais de Tuberculose (SITETB).

Local de estudo

O local de estudo do artigo vigente é o Estado de Pernambuco. Este, possui 185 municípios que são monitorados pelas ações do Programa de Controle de Tuberculose que é responsável pela vigilância epidemiológica da doença. A rede de atenção à saúde ao paciente com TB segue as diretrizes federais: inicia-se na atenção básica, onde é realizado o diagnóstico e seguimento do tratamento 
da doença, podendo ser referenciado para a atenção secundária caso o paciente resida em área descoberta por Equipe da Saúde da Família, ou apresente maiores reações adversas ao uso dos fármacos de tratamento, e ainda se este apresentar comorbidades de difícil manejo como por exemplo o Human Immunodeficiency Virus (HIV). Em caso de TBDR confirmado, o paciente deve ser encaminhado para a referência terciária, que em Pernambuco é constituída pelo Hospital Otávio de Freitas e Hospital das Clínicas, ambos localizados no Município do Recife.

Coleta de dados

Os dados sobre TBDR são provenientes do SITETB, que tem por objetivo registrar a notificação, reunindo informações dos pacientes com TB que necessitem realizar um tratamento diferente do esquema padrão, devido às resistências aos fármacos, reações adversas e/ou certas comorbidades. Dados sobre o total de casos de TB por ano em Pernambuco foram coletados no Sistema de Informações e Agravos de Notificação (SINAN), disponibilizados no Departamento de Informática do Sistema Único de Saúde (DATASUS). Os dados populacionais para cálculo dos coeficientes de incidência (população dos municípios estudados) foram coletados no site do Instituto Brasileiro de Geografia e Estatística (IBGE).

As variáveis analisadas no estudo foram as seguintes:

a) Dependentes: número absoluto de casos de TB-DR notificados, em Pernambuco, no período de 2015 a 2018. b) Independentes - Sociodemográficas: sexo, raça/cor, faixa etária, grau de escolaridade, ocupação e endereço de residência no momento da notificação;

c) Independentes - Padrão de adoecimento: padrão de resistência do bacilo, tipo de entrada e situação de encerramento do tratamento da doença.

O período de estudo foram os anos de 2015 a 2018, que foi selecionado por ter sido o marco inicial da construção do "Plano Nacional pelo Fim da Tuberculose" aprovado em 2014. A fim de identificar o curso epidemiológico da TB-DR após a implementação das estratégias que compõem o plano.

Elegeram-se todos os indivíduos residentes em Pernambuco que foram notificados no período de 2015 a 2018 com diagnóstico de TB-DR. Foram excluídos apenas as notificações de pacientes que apresentavam endereço de residência em outro estado do Brasil.

Realizou-se, inicialmente, a análise epidemiológica descritiva dos casos de TB-DR, considerando a distribuição do número absoluto de casos e seus percentuais de acordo com as variáveis independentes adotadas e ano de estudo. Foi calculada a razão de sexos para incidência de TB-DR. Em seguida, elaboraram-se gráficos para verificar o percentual de pacientes que migraram de um padrão de resistência a determinado fármaco para outro, dentro de um mesmo ano de estudo. Também se elaboraram gráficos de coluna para verificar a distribuição do percentual de casos por sexo, raça/cor, faixa etária, escolaridade e ocupação.

Para análise de tendência do número 
absoluto e proporcional de casos de TB-DR em Pernambuco realizou-se o teste de Shapiro-Wilk para verificação de normalidade, considerando um nível de significância de $5 \%$. Depois, realizou-se o teste de Mann-Kendall para verificar a existência de tendência, utilizando-se, também, uma significância de $5 \%$. Por fim, visando verificar o padrão espacial de distribuição da TB-DR em Pernambuco, foram elaborados mapas temáticos mostrando a variabilidade da incidência desta doença durante os anos de estudo. Aplicou-se o cálculo de coeficiente de incidência para cada município.

Adotou-se a base cartográfica dos municípios do Estado de Pernambuco, em formato shapefile - no sistema de projeção geográfico latitude/longitude e no sistema geodésico de referência SIRGAS 2000, coletada no sítio eletrônico do IBGE. Os dados foram tabulados no programa Excel. Para elaboração dos gráficos de tendência, utilizou-se linguagem $\mathrm{R}$.

Aspectos éticos

Por ser uma análise de dados referentes a seres humanos o projeto foi submetido à Plataforma Brasil respeitando a Resolução 466/12 do Conselho Nacional de Saúde, sendo esta pesquisa proveniente do projeto intitulado "Análise espacial dos casos de tuberculose drogarresistente no Estado de Pernambuco no período de 2015 a 2019" e aprovado pelo comitê de ética do $\mathrm{HUOC} /$ PROCAPE com Certificado de Apresentação de Apreciação Ética (11524819.1.0000.5192).

\section{RESULTADOS}

No período de 2015 a 2018 foram re- gistrados 299 casos de TB-DR no Estado de Pernambuco, com a média de incidência anual de 2,26 casos por 100.000 hab. em 2015; 2,24 em 2016; 2,8 em 2017 e 1,5 em 2018. Com uma razão entre os sexos de aproximadamente 2 homens para cada 1 mulher, sendo o número de homens maior em todo o período, porém acentuado no ano de 2015. Verificou-se uma maior frequência de registros na cor não branca (pardos e negros), compreendendo $242(80,9 \%)$ notificações. Quanto à escolaridade, $175(58,5 \%)$ pessoas possuíam até 7 anos de estudos. O maior número de casos se encontrou na faixa etária de 21 a 30 anos de idade, compondo 77 (25,7\%) das notificações, destacando-se no ano de 2015. O desemprego aponta como atividade laboral mais frequente, com $113(37,7 \%)$ casos (Tabela1).

Quanto ao padrão de adoecimento $115(38,5 \%)$ casos deram entrada no SITETB com resistência à Rifampicina (TB-RR); 113 (37,8\%) com TB-MDR; 51 (17\%) com monorresistência; 15 (5\%) com polirresistência e por fim $5(2 \%)$ deram entrada com TB-XDR. Foram observadas migrações entre os tipos de resistências nos pacientes dentro de cada ano estudado: foram registrados como entrada, predominantemente, casos com resistência apenas à rifampicina, mas, para o mesmo ano, parte deles passaram a apresentar resistência a mais de uma droga, predominando a TB-MDR. Com relação ao tipo de entrada do paciente no SITETB, 210 (70\%) foram notificados como casos novos, e esta categorização destacou-se no ano de 2016. Quanto ao encerramento do acompanhamento do paciente no sistema, prevaleceu a categorização de encerramento por tratamento completo, destacando o ano de 2018 (Tabela 2). 
Tabela 1. Características das variáveis sociodemográficas dos pacientes notificados com tuberculose drogarresistente residentes em Pernambuco. Recife, PE, Brasil

\begin{tabular}{|c|c|c|c|c|c|}
\hline \multicolumn{2}{|c|}{ Variáveis sociodemográficas } & $\begin{array}{l}2015 \\
\mathrm{n}(\%) \\
\end{array}$ & $\begin{array}{l}2016 \\
\mathrm{n}(\%)\end{array}$ & $\begin{array}{l}2017 \\
\text { n (\%) } \\
\end{array}$ & $\begin{array}{l}2018 \\
\mathrm{n}(\%) \\
\end{array}$ \\
\hline \multirow{3}{*}{ Sexo } & Feminino & $21(24)$ & $31(42)$ & $27(37,5)$ & $19(29)$ \\
\hline & Masculino & $66(76)$ & $43(58)$ & $45(62,5)$ & $47(71)$ \\
\hline & Total & $87(100)$ & $74(100)$ & $72(100)$ & $66(100)$ \\
\hline \multirow{4}{*}{ Raça } & Branca & $15(17)$ & $14(19)$ & $13(18)$ & $07(11)$ \\
\hline & Não branca & $70(80)$ & $60(81)$ & $56(78)$ & $56(86)$ \\
\hline & Ignorada & $03(03)$ & - & $03(04)$ & $02(03)$ \\
\hline & Total & $87(100)$ & $74(100)$ & $72(100)$ & $66(100)$ \\
\hline \multirow{6}{*}{ Escolaridade } & 0 & $01(01)$ & $05(07)$ & $06(08)$ & $04(06)$ \\
\hline & $1-7$ & $52(60)$ & $39(53)$ & $37(51)$ & $47(71)$ \\
\hline & $8-11$ & $19(22)$ & $20(27)$ & $19(27)$ & 07 (11) \\
\hline & 12 ou mais & $04(04)$ & $10(13)$ & $03(04)$ & $02(03)$ \\
\hline & Ignorada & $11(13)$ & - & $07(10)$ & $06(09)$ \\
\hline & Total & $87(100)$ & $74(100)$ & $72(100)$ & $66(100)$ \\
\hline \multirow{7}{*}{ Faixa etária } & $15-20$ anos & $02(02)$ & $02(03)$ & $03(04)$ & $07(10)$ \\
\hline & $21-30$ anos & $22(26)$ & $21(28)$ & $19(26)$ & $15(23)$ \\
\hline & $31-40$ anos & $22(26)$ & $18(25)$ & $17(25)$ & $13(20)$ \\
\hline & $41-50$ anos & $17(20)$ & $12(16)$ & $19(26)$ & $16(24)$ \\
\hline & $51-60$ anos & $17(20)$ & $11(15)$ & $10(14)$ & $09(14)$ \\
\hline & $>60$ anos & $06(06)$ & $10(13)$ & $04(05)$ & $06(09)$ \\
\hline & Total & $87(100)$ & $74(100)$ & $72(100)$ & $66(100)$ \\
\hline \multirow{9}{*}{$\begin{array}{l}\text { Ocupação/ } \\
\text { Atividade } \\
\text { laboral }\end{array}$} & Autônomos & $33(38)$ & $20(27)$ & $15(21)$ & $09(13)$ \\
\hline & Aposentados & $05(06)$ & $06(08)$ & $09(12)$ & $04(06)$ \\
\hline & Desempregado & $23(27)$ & $30(40)$ & $32(45)$ & $28(42)$ \\
\hline & Estudantes & $01(01)$ & $04(05)$ & $04(05)$ & $04(06)$ \\
\hline & Profissional de saúde. & $02(02)$ & $02(03)$ & - & $01(02)$ \\
\hline & $\begin{array}{l}\text { Pop. privada de } \\
\text { liberdade. }\end{array}$ & $11(13)$ & $08(11)$ & $05(07)$ & $05(08)$ \\
\hline & Pop. de Rua. & $06(06)$ & $01(02)$ & - & $01(02)$ \\
\hline & Ignorada & $07(07)$ & $03(04)$ & $07(10)$ & $14(21)$ \\
\hline & Total & $87(100)$ & $74(100)$ & $72(100)$ & $66(100)$ \\
\hline
\end{tabular}

Em cada ano considerado, houve variação da proporção de pacientes de TB-DR do padrão de monorresistência (no início do ano) para resistência a mais de um fármaco (no fim do ano). Sendo assim, em todos os anos, a maioria dos pacientes que deram entrada no SITETB apresentaram monorresistência, porém foram adquirindo resistência a mais de um fármaco com o passar do tempo. Destacando-se o ano de 2018 com o maior número de casos de migração entre os padrões de resistência: no início do ano, $62 \%$ dos pacientes eram resistentes a apenas um fármaco, e no fim deste mesmo ano esse percentual reduziu para 36\% (Figura 1).

Com relação a distribuição percentual dos casos de TB-DR segundo as variáveis: sexo, raça/cor, escolaridade, faixa etária e ocupação por semestre no período do estudo, percebe-se para todos os períodos um maior percentual de homens não brancos, com escolaridade entre 5 e 7 anos de estudo, com idade 21 a 59 anos e desempregados (Figura 2).

Segundo a evolução temporal do número absoluto de casos de TB-DR em Pernambuco e da proporção de casos de TB-DR 
Tabela 2. Padrão de adoecimento dos pacientes notificados com tuberculose drogarresistente residentes em Pernambuco. Recife, PE, Brasil

\begin{tabular}{|c|c|c|c|c|c|c|c|c|}
\hline \multirow{2}{*}{\multicolumn{2}{|c|}{ Padrão de adoecimento }} & $\begin{array}{r}2015 \\
n(\%) \\
\end{array}$ & \multicolumn{2}{|c|}{$\begin{array}{l}2016 \\
\mathrm{n}(\%) \\
\end{array}$} & \multicolumn{2}{|c|}{$\begin{array}{l}2017 \\
\mathrm{n}(\%) \\
\end{array}$} & \multicolumn{2}{|c|}{$\begin{array}{l}2018 \\
\mathrm{n}(\%) \\
\end{array}$} \\
\hline & & Inicial final & Inicial & Atual & Inicial & Atual & Inicial & Atual \\
\hline \multirow{6}{*}{$\begin{array}{l}\text { Padrão de } \\
\text { Resistência }\end{array}$} & Resistência a rifampicina & $24(28) 19(22)$ & $29(39)$ & $23(31)$ & $31(43)$ & $15(21)$ & $31(47)$ & $10(15)$ \\
\hline & Monorresistente & $17(19) 19(22)$ & 08 (11) & $08(11)$ & $16(22)$ & $23(32)$ & $10(15)$ & $14(22)$ \\
\hline & Polirresistente & $05(06) 05(06)$ & $07(09)$ & $07(09)$ & $01(01)$ & $02(03)$ & $02(03)$ & $03(04)$ \\
\hline & Multidrogarresistente & 38 (44) 41 (47) & $29(39)$ & $34(46)$ & $23(33)$ & $31(43)$ & $23(35)$ & $38(58)$ \\
\hline & $\begin{array}{l}\text { Extensi } \\
\text { resister }\end{array}$ & $03(03) 03(03)$ & $01(02)$ & $02(03)$ & $01(01)$ & $01(01)$ & - & $01(01)$ \\
\hline & Total & \multicolumn{3}{|c|}{$87(100) 87(100) 74(100) 74(100)$} & \multicolumn{2}{|c|}{$72(100) 72(100)$} & $66(100)$ & $66(100)$ \\
\hline \multirow{3}{*}{$\begin{array}{l}\text { Entrada no } \\
\text { SITETB }\end{array}$} & $\begin{array}{l}\text { Caso novo } \\
\text { Falência ao retratamento } \\
\text { Retorno após abandono }\end{array}$ & $\begin{array}{l}55(63) \\
08(09) \\
14(17)\end{array}$ & \multicolumn{2}{|c|}{$\begin{array}{l}57(77) \\
04(05) \\
12(16)\end{array}$} & \multicolumn{2}{|c|}{$\begin{array}{l}51(71) \\
03(04) \\
10(14)\end{array}$} & \multicolumn{2}{|c|}{$\begin{array}{l}47(71) \\
03(05) \\
14(21)\end{array}$} \\
\hline & Falência ao $1^{\circ}$ & $07(08)$ & \multicolumn{2}{|c|}{$01(02)$} & \multicolumn{2}{|c|}{$05(07)$} & \multicolumn{2}{|c|}{ - } \\
\hline & $\begin{array}{l}\text { Recidiva } \\
\text { Total }\end{array}$ & $\begin{array}{c}02(03) \\
87(100)\end{array}$ & & \multicolumn{2}{|c|}{$\begin{array}{c}03(04) \\
72(100)\end{array}$} & \multicolumn{2}{|c|}{$\begin{array}{c}02(3) \\
66(100)\end{array}$} \\
\hline \multirow{7}{*}{$\begin{array}{l}\text { Situação de } \\
\text { encerramento }\end{array}$} & Tratamento Completo & $21(24)$ & \multicolumn{2}{|c|}{$30(40)$} & \multicolumn{2}{|c|}{$22(30)$} & \multicolumn{2}{|c|}{$02(03)$} \\
\hline & Abandono & $29(33)$ & \multicolumn{2}{|c|}{$20(27)$} & \multicolumn{2}{|c|}{$23(32)$} & \multicolumn{2}{|c|}{$17(26)$} \\
\hline & Obito & \multirow{2}{*}{$\begin{array}{l}13(15) \\
13(15)\end{array}$} & \multirow{2}{*}{\multicolumn{2}{|c|}{$\begin{array}{l}09(12) \\
08(11)\end{array}$}} & \multirow{2}{*}{\multicolumn{2}{|c|}{$\begin{array}{l}12(17) \\
03(04)\end{array}$}} & \multirow{2}{*}{\multicolumn{2}{|c|}{$\begin{array}{l}07(11) \\
02(03)\end{array}$}} \\
\hline & Curado & & & & & & & \\
\hline & Falência & $11(13)$ & 05 ( & 07) & 03 & 04) & & - \\
\hline & Em tra & - & 02( & 03) & & 13) & 38 & (57) \\
\hline & Total & $87(100)$ & $74(1)+1)(x)+2)$ & 100) & 72( & 100) & 66( & $(100)$ \\
\hline
\end{tabular}

Figura 1. Variação da proporção de pacientes de TB diagnosticados com drogarresistência em Pernambuco, Recife, PE, Brasil
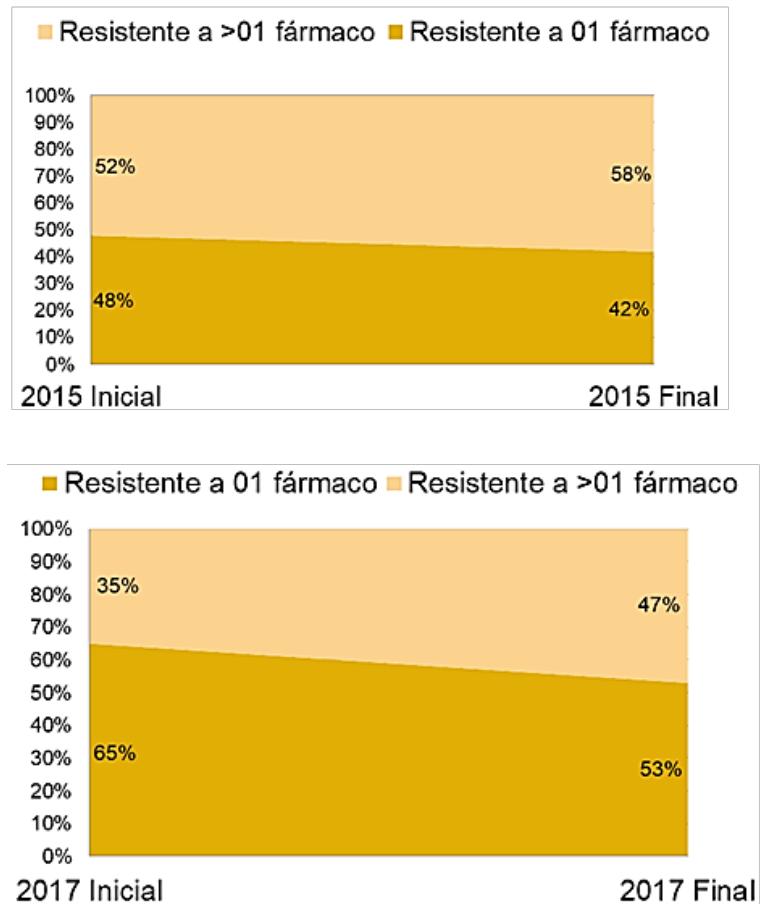

Resistente a >01 fármaco $\square$ Resistente a 01 fármaco

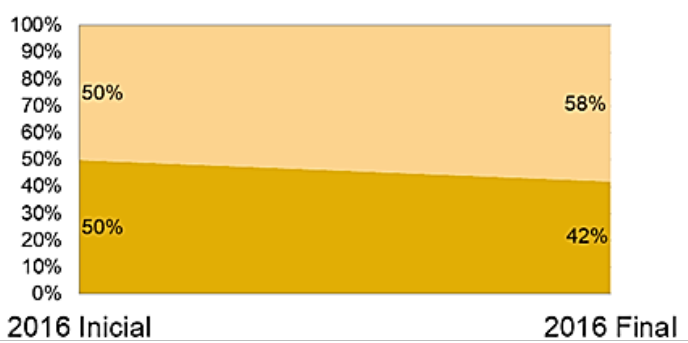

$\llbracket$ Resistente $a>01$ fármaco $₫$ Resistente a 01 fármaco

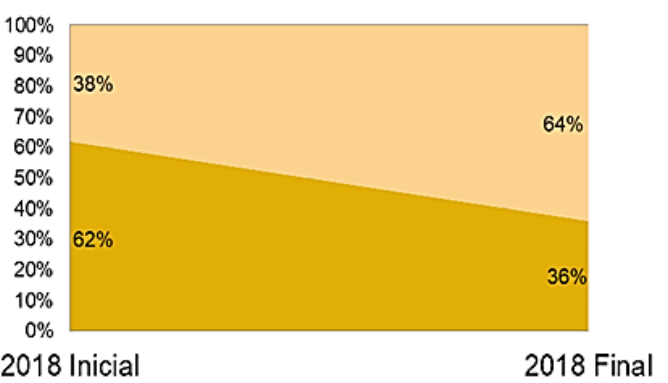


com relação aos demais casos de TB no estado, o ajuste do modelo linear para os casos de TB revelou um coeficiente angular de $-0,42$ com valor-p de 0,38 (para $p<0,05$ ), indicando uma tendência não significativa, mostrando que a distribuição dos casos permanece a mesma com o tempo. Também foi aplicado o teste de Mann-Kendall, que mostrou igualmente que não existiu tendência significativa (valor- $p=0,78 ; p<0,05$ ). Também se realizou ajuste do modelo linear para a proporção de casos de TB-DR com relação ao total de casos de TB, resultando em um coeficiente angular igual a $-0,18$ (para $p<0,05$ ), indicando uma tendência não significativa. Ou seja, a distribuição da proporção de casos não muda com o tempo. Ao aplicar o teste de Mann-Kendall, obteve-se um valor de $p$ de 0,39 (para $p<$ 0,05 ) indicando, igualmente, a inexistência de tendência (Figura 3).

Em relação a distribuição espacial dos coeficientes de incidência de TB-DR no Estado de Pernambuco, verificou-se que, ao longo dos quatro anos analisados, o maior número de municípios com casos de TB-DR no Estado de Pernambuco concentrou-se na Região Metropolitana do Recife (RMR). Os maiores coeficientes de incidência entre 2015 e 2018 por 100.000 habitantes foram registrados na Ilha de Itamaracá $(4,77)$, Buenos Aires $(3,80)$, Gameleira $(3,2)$, Olinda $(2,68)$, Tamandaré $(2,14)$, Cabo de Santo Agostinho $(2,05)$ e Recife $(2,05)$, sendo que destes, somente Buenos Aires e Gameleira não fazem parte

Figura 2. Distribuição percentual dos casos de tuberculose drogarresistente segundo o sexo, a raça/ cor, a escolaridade, a faixa etária e a ocupação. Recife, PE, Brasil

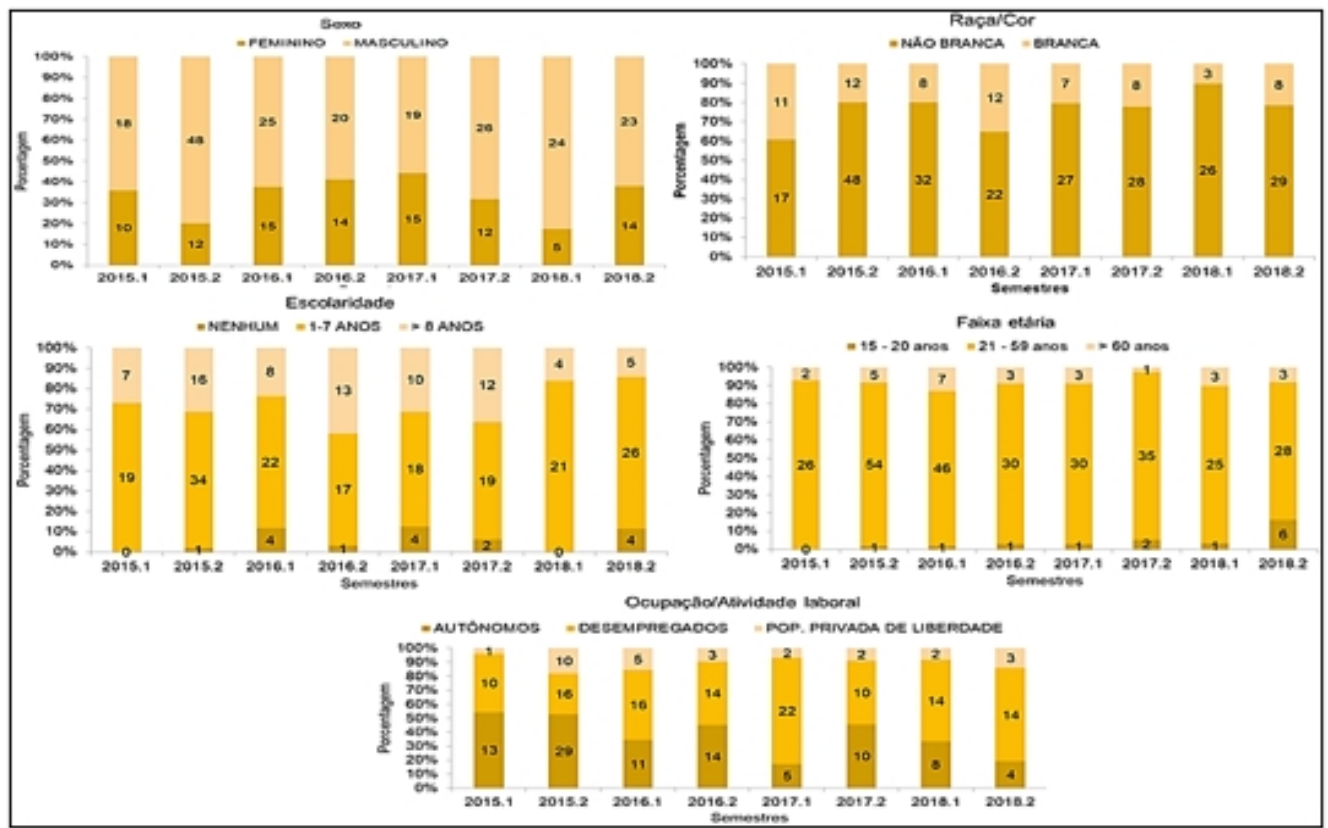


Figura 3. Evolução temporal do número absoluto de casos de tuberculose drogarresistente e de sua proporção em relação ao total de casos de tuberculose em Pernambuco. Recife, PE, Brasil
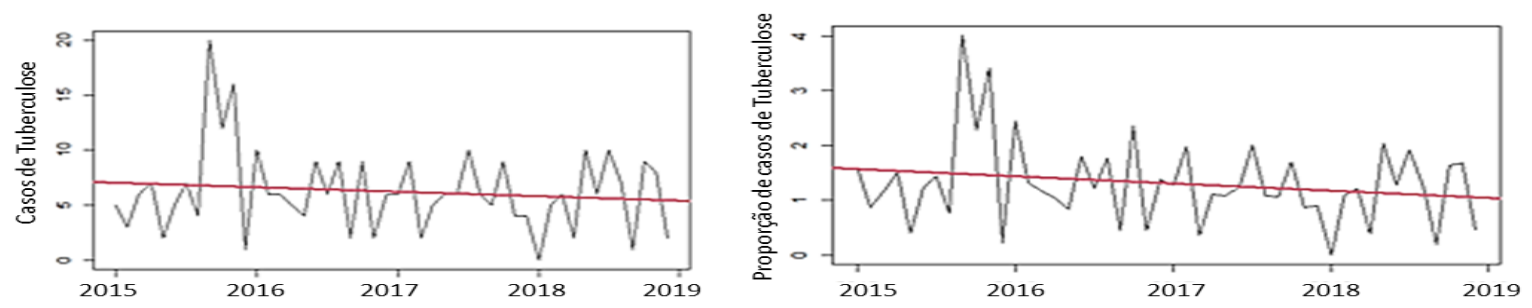

Figura 4. Distribuição espacial da incidência de tuberculose drogarresistente (por 100 mil habitantes) nos municípios do estado de Pernambuco. Recife, PE, Brasil

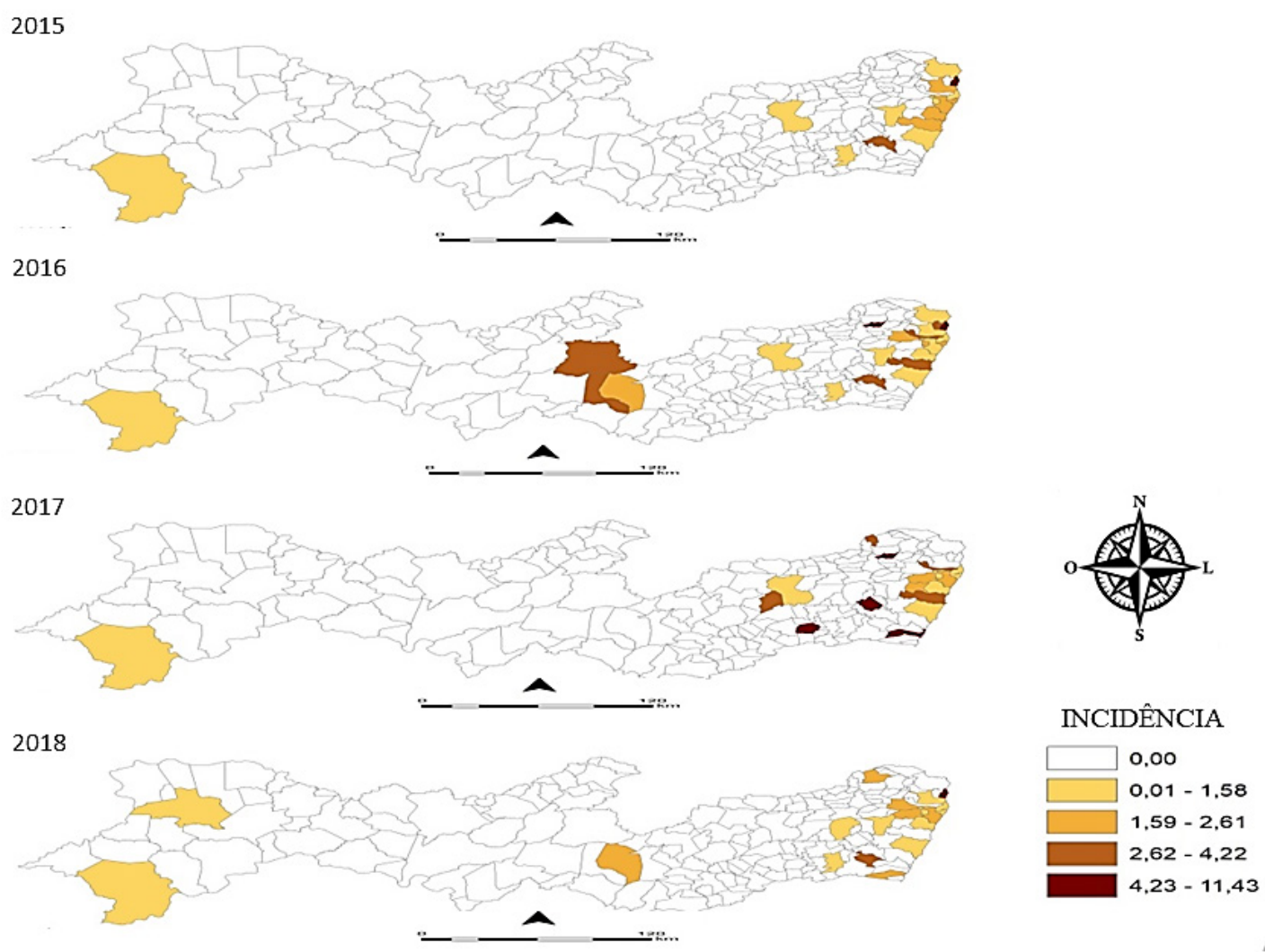

da Região Metropolitana de Recife. Quanto ao número absoluto de casos, têm-se a Capital Recife com (118) casos, Jaboatão dos Guararapes com (39), Olinda (17), Cabo de
Santo Agostinho (13), Paulista e Abreu e Lima com (7) casos de TB-DR, todos localizados na RMR (Figura 4). 


\section{DISCUSSÃO}

O estudo analisou as características clínicas e epidemiológicas, bem como a evolução temporal e a distribuição espacial dos casos de TB-DR no Estado Pernambucano. Observou-se predominância do sexo masculino, de cor não branca, adultos jovens (21 e 30 anos), desempregados com menos de 7 anos de estudos, resistentes inicialmente à rifampicina e posteriormente multirresistentes, classificados como casos novos e localizados em municípios situados na região metropolitana do estado. Viu-se, também, que não ocorreu tendência à redução nem ao aumento do número de casos com o tempo e que a maioria dos casos se concentrou na RMR.

O sexo masculino destacou-se por concentrar o maior número de casos neste estudo, com cerca de $67 \%$ do total de casos. Estes achados corroboram com um estudo realizado no Rio de Janeiro que investigou os fatores associados à interrupção e óbitos no curso de tratamento de TB-DR, entre 2012 e 2013, identificando o sexo masculino como a maioria da população de estudo. Este fato pode estar relacionado aos hábitos de vida do sexo masculino, que tem maior propensão ao consumo de álcool com consequentes e contínuas interrupções e/ou abandonos de tratamentos clínicos; como também a pouca adesão à procura dos serviços de saúde o que acarreta diagnósticos tardios ${ }^{16,17}$.

O maior percentual de casos de TB-DR foi na população não branca, semelhante ao perfil predominante nos pacientes com TB sensível, observados em estudo realizado no Maranhão, que investigou os fatores associados ao retratamento por recidiva e por reingresso após abandono, no período entre 2005 e 2010 17 . Por outro lado, um estudo realizado em Santa Catarina por Costa et al. ${ }^{18}$, que traçou o perfil epidemiológico dos pacientes com TB-DR, identificou predominância da cor branca nos pacientes. Este fato, contudo, pode ser explicado por este estado localizar-se na Região Sul do país. Ainda assim, para o Brasil como um todo, o fato de se encontrar predominância de casos de TB-DR na população não branca pode estar associado ao fato de que este contingente populacional está inserido em piores contextos sociais. Contudo, destaca-se que, mesmo que o perfil dos pacientes com TB-DR seja predominantemente de negros e pardos, não pode ser descartada a suscetibilidade geral da doença na população, devendo ser considerado também o padrão racial encontrado em cada território ${ }^{18,19}$.

O maior número de casos entre pessoas com baixa escolaridade, encontrado neste estudo, coincide com resultado encontrado no Rio de Janeiro no período entre 2012 e 2013 sobre interrupção de tratamento da TB-DR. Este trabalho associou o baixo nível de educação ao aumento da vulnerabilidade à $T B$, pois reflete o acesso individual e desigual à informação, a benefícios gerais que advém do conhecimento, aos bens de consumo e ao serviço de saúde. Some-se a isso que a baixa escolarização favorece o abandono, a falha medicamentosa e, finalmente, o agravo da doença ou óbito ${ }^{16}$.

Encontrou-se predominância de adul- 
tos jovens entre 21 e 30 anos de idade. Tal achado diverge do estudo realizado no Rio de Janeiro e do estudo em Santa Catarina no período de 2010 a 2015, que mesmo identificando predominância da TB-DR na população jovem, o foco encontrado é na faixa etária entre 30 e 49 anos. Segundo estes estudos, existe uma relação entre a juventude e a taxa de abandono ao tratamento da doença, por questões de hábitos individuais relacionados à imaturidade e ao não entendimento sobre a importância do tratamento. Destaca-se que a existência de jovens adultos bacilíferos indica a ocorrência de transmissão ativa da doença ${ }^{16-18}$.

Quanto à situação ocupacional, a maioria dos casos se distribuíram entre pessoas com nenhuma ocupação/atividade laboral. Estudos que investigaram fatores associados à incidência de TB e de TB-DR na população brasileira, nos anos de 2010 e 2014 demonstraram que o aumento do desemprego no país nos últimos anos acompanhou-se de piores condições de vida, que favorece o acometimento pela doença com consequente evasão do trabalho. Isso reproduz um ciclo, onde as más condições de vida levam ao adoecimento, assim como este produz pobreza, quando limita as oportunidades de subsistência ${ }^{4,13,20}$.

Ocorreram mudanças no perfil de resistência do bacilo ao longo do tratamento dos pacientes dentro de um mesmo ano. Observou-se que, no momento da entrada no sistema, a maioria apresentava resistência a um único fármaco, ao passo que no fim do mesmo ano, tinham migrado para resistente a mais de uma droga. Um estudo realizado com cepas isoladas de pacientes no Peru discute essa mudança da resistência, associando a sistemas de compensação da própria bactéria que geram mutações em seu genoma ${ }^{21}$.

Durante o período estudado, a maioria dos pacientes notificados deram entrada no SITETB como caso novo e foram encerrados com abandono de tratamento. Este fato se relaciona com a manutenção ativa de transmissão da doença, o que se agrava com este abandono do tratamento. Esse achado não é atípico, pois assemelha-se com o resultado do estudo de Costa et al. ${ }^{18}$ onde o percentual de casos novos foi cerca de $93 \%$ da sua amostra no Estado de Santa Catarina. Outros resultados apontam o nível de escolaridade como um dos fatores principais de abandono de tratamento da doença ${ }^{12,16,18}$.

Se, por um lado, fala-se das características individuais próprias ou do bacilo que propiciam a manutenção dos padrões de resistência em determinado local, por outro, faz-se necessário abordar o papel dos serviços de saúde. Estudo realizado em 2020, em Recife, que visou avaliar a coordenação do cuidado entre níveis de atenção ao paciente com TB, mostrou que embora venham sendo reforçadas há certo tempo a descentralização das ações de diagnóstico e tratamento para as unidades de atenção básica, ainda existem fragilidades na atenção ao doente de TB, constituindo um obstáculo na garantia da integralidade, do acesso e da oferta de serviços de saúde e até na interrupção do tratamento ${ }^{11,22}$.

Esta afirmativa pode ser considerada quando se observa a evolução temporal dos 
casos de TB-DR em Pernambuco. Os resultados deste estudo mostram que, mesmo que medidas específicas tenham sido adotadas pelo estado, existe tendência à manutenção do número de casos de TB-DR e da proporção destes com relação a todos os casos de $\mathrm{TB}^{8,7}$.

O elevado número de casos de TB e de TB-DR em particular na Região Nordeste tem apresentado um constante crescimento populacional nas áreas urbanas, principalmente nas capitais e nas conurbações. Este fato pode ser explicado pela urbanização desordenada, que favorece a aglomeração de pessoas em condições insalubres de habitação que, juntamente com outros fatores como a má nutrição, aumentam os bolsões de pobreza e favorecem a disseminação e a manutenção do ciclo de transmissão desta doença. Este fato, somados aos fatores individuais, favorece o aumento da resistência medicamentosa à $\mathrm{TB}^{23}$.

O fato dos casos de TB-DR estarem concentrados na região metropolitana e serem poucos os identificados no interior do Estado, também pode estar relacionado à organização do território que costuma ser menos populoso e com isso implicar em baixa transmissibilidade da doença. Outro fator que pode estar associado à concentração de casos de TB-DR na RMR pode ser a subnotificação ocorrida no interior. Estudo realizado em Pernambuco, em 2018, visando verificar os fatores relacionados à subnotificação de TB em PE pode reforçar esta hipótese, pois identificou que as chances de subnotificação eram maiores para aquelas pessoas provenientes do interior ${ }^{24}$. As limitações do estudo referem-se à utilização de dados secundários, que devido a incompletude ou preenchimento equivocado dos dados, são passíveis a vieses nos resultados deste estudo.

\section{CONCLUSÃO}

Neste estudo, as características sociodemográficas dos casos de TB-DR encontradas, foram semelhantes às vistas na literatura, observando-se a predominância do sexo masculino, cor não branca, faixa etária de 21 a 30 anos, desempregados com menos de 7 anos de estudos, resistentes no início à rifampicina com posterior multirresistência e classificados como casos novos. Estes fatores, conjugados com a urbanização desordenada, principalmente na RMR, e com deficiências nos serviços de saúde podem contribuir para que haja tendência de manutenção do número de casos no período do estudo e para a concentração de casos na RMR. Estes achados podem subsidiar outros estudos mais amplos em Pernambuco e em outros contextos, a fim de gerar intervenções em diversas áreas como saúde, educação, políticas, sociais e econômicas que permitam contribuir com o controle da TB e TB-DR e ainda assim, subsidiar ações de vigilância epidemiológica.

\section{REFERÊNCIAS}

1. Nemes E, Geldenhuys H, Rozot V, et al., C-040-404 Study Team. Prevention of M. tuberculosis Infection with H4:IC31 Vaccine or BCG Revaccination. N Engl J Med.2018; 379(2):138-149.

2. Silva DR, Muñoz-Torrico M, Duarte R, et al., Risk factors for tuberculosis: diabetes, smoking, alcohol use, and the use of other drugs. J Bras Pneumol.2018;44(2):45-152. 
3. Hino P, Ferreira AMR, Souza TKMJ, et al., Tuberculosis control from the perspective of health professionals working in street clinics. Rev. Latino-Am. Enfermagem. 2018; 26:e3095.

4. Pelissari DM, Diaz-Quijano FA. Household crowding as a potential mediator of socioeconomic determinants of tuberculosis incidence in Brazil. PLoS One. 2017; 12(4):e0176116.

5. Teixeira AQ. et al., Tuberculose: conhecimento e adesão às medidas profiláticas em indivíduos contatos da cidade do Recife, Pernambuco, Brasil. Cad. Saúde Colet. 2020; 28(1): 116-129.

6. Brasil. Ministério da Saúde. Secretaria de Vigilância em Saúde. Departamento de Vigilância das Doenças Transmissíveis. Manual de Recomendações para o Controle da Tuberculose no Brasil - Brasília: Ministério da Saúde, 2019.

7. Brasil. Ministério da Saúde. Secretaria de Vigilância em Saúde. Departamento de Vigilância das Doenças Transmissíveis. Brasil Livre da Tuberculose: Plano Nacional pelo Fim da Tuberculose como Problema de Saúde Pública - Brasília : Ministério da Saúde, 2017.

8. Barreira D. The challenges to eliminating tuberculosis in Brazil. Epidemiol Serv Saude. 2018; 27(1):e00100009.

9. Kritski A, Dalcolmo MP, Mello CQ, et al., The role of the Brazilian Tuberculosis Research Network in national and international efforts to eliminate tuberculosis. J Bras Pneumol. 2018. 44(2):77-81.

10. Cecilio HPM, Santos AL, Marcon SS, et al., Tuberculosis mortality trend in the state of Paraná, Brazil - 1998-2012. Cien Saude Colet. 2018; 23(1):241-248.

11. Barros DBM, Costa JMBS, Reis YAC, et al., Evaluation of care coordination of users with multi-drug tuberculosis in the city of Recife, Pernambuco State, Brazil. Saúde Debate. 2020; 44(124):99-114.

12. Fiorati RC, Cãndido FCA, Souza LB, et al., Desigualdades sociais e os desafios à estratégia de eliminação da tuberculose no Brasil [Internet]. Vittalle - Revista de Ciências da Saúde. 2018; 30(2):59-72.

13. Jacobs MG. Tuberculose drogarresistente no Brasil: perfil de casos, distribuição territorial e fatores associados. 2017. [Dissertação]Universidade de Brasília, Brasília, 2017.
14. Ferreira AB. Avaliação de impacto da estratégia TDO no controle da tuberculose em Pernambuco. 2019. [Tese] Universidade Federal de Pernambuco, 2017.

15. Jacobs MG, Pinto Junior VL. Brazilian cities profile, the occurence of tuberculosis an its drug-resistant form. Cien Saude Colet. 2019. 22;24(7):2379-2386.

16. Viana PVS, Redner P, Ramos JP, et al., Factors associated with loss to follow-up and death in cases of drug-resistant tuberculosis (DR-TB) treated at a reference center in Rio de Janeiro, Brazil. Cad Saude Publica. 2018; 34(5):e00048217.

17. Silva TC, Silva PF, Dornele M, et al., Factors associated with tuberculosis retreatment in priority districts of Maranhão, Brazil. Cien Saude Colet. 2017; 2(12):4095-4103.

18. Costa YHM. et al., Tuberculose drogarresistente em Santa Catarina no período de 2010 a 2015: pacientes curados. Revista AMRIGS, Porto Alegre, 2018.

19. Ceccon RF, Maffacciolli R, Burille A, et al., Tuberculosis mortality in Brazilian capitals, 2008-2010.Epidemiol Serv Saude. 2017; 26(2):349-358.

20. Pedro AS, Jefferson GG, Santos PC, et al., Tuberculosis as a marker of inequities in the context of socio-spatial transformation. Rev Saude Publica. 2017; 51:9

21. Vargas AP, Rios AA, Grandjean L, et al., Determination of potentially novel compensatory mutations in rpoc associated with rifampin resistance and rpob mutations in Mycobacterium tuberculosis Clinical isolates from peru. Int J Mycobacteriol. 2020; 9 (2): 121-137.

22. Jacobs MG, Pinto Junior VL. Characterization of drug-resistant tuberculosis in Brazil, 2014. Epidemiol Serv Saude. 2019; 28(3):e2018294.

23. Queiroz AAR, Berra TZ, Garcia CC, et al., Spatial pattern and temporal trend of mortality due to tuberculosis. Rev. Latino-Am. Enfermagem. 2018; 26:e2992.

24. Santos ML, oeli CM, Batista JDL, et al., Factors associated with underreporting of tuberculosis based on data from Sinan Aids and Sinan TB. Rev Bras Epidemiol. 2018; 21: e180019.

25. Jaime EGD. et al., Political determinants of Heath: a concept of importance for the professional in public health. Novel Opinion - Rev. avances en salud (Montería. En línea). 2019. 
CORRESPONDÊNCIA

Karla Naiara França Silva

karlafrancca@gmail.com 